\title{
Static properties of a quasi one-dimensional antiferromagnet in magnetic field
}

\author{
M. E. Zhitomirsky \\ Institute for Solid State Physics, University of Tokyo, Tokyo 106, Japan \\ and L. D. Landau Institute for Theoretical Physics, Moscow 117334 Russia \\ I. A. Zaliznyak \\ P. L. Kapitza Institute for Physical Problems, Moscow 117334, Russia \\ and DRFMC-Centre d'Etudes Nucléaires de Grenoble, BP 85X, 38041 Grenoble Cédex, France
}

(11 October, 1995)

\begin{abstract}
We calculate the effect of zero-point fluctuations on the magnetostatic properties of a quasi one-dimensional antiferromagnetic helimagnet with and without in-plane anisotropy. We find substantial reduction of the uniform susceptibility and the sublattice magnetization from their classical values and appreciable renormalization of the spin-flop field at $T=0$. The magnetization curve varies nonlinearly at small fields and is described by a universal formula at high fields. The results are compared with numerical simulations on one-dimensional systems and measurements in the $\mathrm{CsNiCl}_{3}$-type compounds.
\end{abstract}

PACS numbers: 75.10Jm, 75.50Ee, 75.60.Ej

Typeset using REVTEX 


\section{INTRODUCTION}

Recently, there has been growing interest in the behavior of one-dimensional and quasi one-dimensional antiferromagnets in strong magnetic fields. In the former case much attention was devoted to the destruction of the Haldane state of a spin-1 antiferromagnetic chain in applied field and to the magnetization process at higher fields [1-7]. In the latter case the interest lies mainly in properties of $\mathrm{CsNiCl}_{3}$-type magnetic compounds which are good model Heisenberg systems. They contain a stacked triangular lattice of antiferromagnetically coupled $3 d$ ions with the intrachain exchange constant $J$ being several orders of magnitude larger than the interchain coupling $J^{\prime}$. Because of such smallness of interchain interactions, quantum effects play an important role in the Néel phase. Extensive theoretical works have been presented to describe quantum renormalizations of the magnon spectrum at $T=0$ in these materials [9-11]. Though experiments indicate significant deviations in static properties of quasi one-dimensional antiferromagnets from predictions of the classical theory as well [12-18], only a few effects were considered theoretically $[11,19,20]$.

Here we study the lowest order quantum corrections to the static characteristics of a quasi one-dimensional antiferromagnetic helimagnet: staggered magnetization, transverse and inplane magnetic susceptibilities, spin-flop field, and full magnetization curve. We show that the magnetization process at high fields is the same for all low-dimensional systems, i.e., is independent from the nature of the ground state at $H=0$. This fact is connected with the different corrections to the staggered and uniform magnetizations appearing in the spin-wave theory.

The model is defined by the Heisenberg Hamiltonian for a system of $N$ equivalent spins on a simple Bravais lattice with a single-ion anisotropy and the Zeeman term:

$$
\hat{\mathcal{H}}=\sum_{i, j} J_{i j} \mathbf{S}_{i} \cdot \mathbf{S}_{j}+D \sum_{i}\left(S_{i}^{z}\right)^{2}-\sum_{i} \gamma \mathbf{H} \cdot \mathbf{S}_{i}
$$

(in the following, we include the gyromagnetic ratio $\gamma=g \mu_{B}$ in the definition of $H$ ). Without anisotropy and magnetic field the classical ground state of (1) is a plane spin helix $\mathbf{S}_{i}=\left(S \cos \theta_{i}, S \sin \theta_{i}, 0\right), \theta_{i}=\mathbf{Q} \cdot \mathbf{r}_{i}$, where the ordering wave vector $\mathbf{Q}$ corresponds to the minimum of the Fourier transform of exchange interactions: $J_{\mathbf{k}}=\sum_{i} J_{i} \exp \left(i \mathbf{k} \cdot \mathbf{r}_{i}\right)[21]$.

To obtain the first $1 / S$ correction to the classical energy of the ordered state we use the standard perturbation scheme in which spin operators are replaced by their bosonic equivalents by applying the reduced Holstein-Primakoff transformation $S^{z}=S-a^{+} a, S^{+}=\sqrt{2 S} a$, 
$S^{-}=\sqrt{2 S} a^{+}$to each spin in its local coordinate system. The first order corrections appear in this harmonic approximation due to the quadratic part of the boson Hamiltonian which corresponds to the quantized classical spin waves with zero-point oscillations. Diagonalization of the boson Hamiltonian is trivial for a helimagnet without in-plane anisotropy $(D>0, \mathbf{H} \| \hat{\mathbf{z}})$. Analytical expressions for quantum effects under these conditions are given in Sec. II. Less studied in the literature case of a bunched spiral in the presence of an in-plane anisotropy is considered in Sec. III. Treating perturbatively helix distortions we calculate the classical spin-wave spectrum and find the quantum effects for a spin helix with the easy-axis anisotropy and in-plane magnetic field. Comparison of the theory with experimental measurements in $\mathrm{CsNiCl}_{3}$ and $\mathrm{CsMnBr}_{3}$ is presented in Sec. IV.

\section{AXIALLY SYMMETRIC CASE}

We first present standard spin-wave calculations for a helimagnet with the easy-plane type of anisotropy $(D>0)$ and magnetic field parallel to the symmetry axis $\hat{\mathbf{z}}$ (see, e.g., Ref. [21]) and find analytical expressions for quantum effects in the quasi onedimensional limit. For this axially symmetric case spins form an umbrella structure $\mathbf{S}_{i}=\left(S \cos \alpha \cos \theta_{i}, S \cos \alpha \sin \theta_{i}, S \sin \alpha\right)$, where $\theta_{i}=\mathbf{Q} \cdot \mathbf{r}_{i}$. By minimizing the classical energy of the deformed spin helix one obtains an expression for the canting angle $\sin \alpha=H / H_{s}$, which is valid up to the saturation field $H_{s}=2\left(J_{0}-J_{\mathbf{Q}}+D\right) S$. Above this critical field $\sin \alpha=1$ and all spins are aligned parallel to $\mathbf{H}$.

The quadratic part of the Hamiltonian after the normal ordering and Fourier transformation of boson operators is written in the form:

$$
\hat{\mathcal{H}}_{2}=\frac{1}{2} N D S \cos ^{2} \alpha+\sum_{\mathbf{k}}\left[\left(A_{\mathbf{k}}+C_{\mathbf{k}}\right) a_{\mathbf{k}}^{+} a_{\mathbf{k}}+\frac{1}{2} B_{\mathbf{k}}\left(a_{\mathbf{k}} a_{-\mathbf{k}}+a_{\mathbf{k}}^{+} a_{-\mathbf{k}}^{+}\right)\right],
$$

with

$$
\begin{aligned}
& A_{\mathbf{k}}=-2 S J_{\mathbf{Q}}+S \cos ^{2} \alpha\left(J_{\mathbf{k}}+D\right)+\frac{1}{2} S\left(1+\sin ^{2} \alpha\right)\left(J_{\mathbf{k}+\mathbf{Q}}+J_{\mathbf{k}-\mathbf{Q}}\right), \\
& B_{\mathbf{k}}=-S \cos ^{2} \alpha\left[J_{\mathbf{k}}+D-\frac{1}{2}\left(J_{\mathbf{k}+\mathbf{Q}}+J_{\mathbf{k}-\mathbf{Q}}\right)\right], \quad C_{\mathbf{k}}=S \sin \alpha\left(J_{\mathbf{k}+\mathbf{Q}}-J_{\mathbf{k}-\mathbf{Q}}\right) .
\end{aligned}
$$

Note, that we work in the extended zone scheme assigning wave vectors to the paramagnetic Brillouin zone. Coefficients $A_{\mathbf{k}}$ and $B_{\mathbf{k}}$ are even functions of $\mathbf{k}$, while $C_{\mathbf{k}}$ is an odd function. Performing the standard Bogolyubov transformation, which leaves odd-k terms unchanged, one can diagonalize the bilinear form of boson operators: 


$$
\sum_{\mathbf{k}}\left[\omega_{\mathbf{k}}\left(b_{\mathbf{k}}^{+} b_{\mathbf{k}}+\frac{1}{2}\right)-\frac{1}{2} A_{\mathbf{k}}\right]
$$

where the classical spin-wave frequencies are given by

$$
\omega_{\mathbf{k}}=\sqrt{A_{\mathbf{k}}^{2}-B_{\mathbf{k}}^{2}}+C_{\mathbf{k}} .
$$

The easy-plane anisotropy creates gaps at $\mathbf{k}= \pm \mathbf{Q}$, whereas spin waves at $\mathbf{k} \rightarrow 0$ remain to be the Goldstone modes up to the saturation field.

We calculate the magnetization at $T=0$ taking the derivative of the ground state energy with respect to the magnetic field

$$
M=N \frac{H}{2\left(J_{0}-J_{\mathbf{Q}}+D\right)}\left[1-\frac{1}{2 S N} \sum_{\mathbf{k}} \frac{\frac{1}{2}\left(J_{\mathbf{k}+\mathbf{Q}}+J_{\mathbf{k}-\mathbf{Q}}\right)-J_{\mathbf{k}}-D}{J_{0}-J_{\mathbf{Q}}+D} \sqrt{\frac{A_{\mathbf{k}}+B_{\mathbf{k}}}{A_{\mathbf{k}}-B_{\mathbf{k}}}}\right] .
$$

The factor before brackets is the magnetization value in the classical theory which is renormalized due to zero-point oscillations. The expression (5) corresponds to the field region $0<H<H_{s}$, whereas in the completely aligned phase above $H_{s}$ quantum corrections disappear and magnetization saturates: $M_{s}=N S$. The average value of the local spin below $H_{s}$ is given by the standard formula [21]

$$
S_{\mathrm{av}}=S\left[1-\frac{1}{2 S N} \sum_{\mathbf{k}}\left(\frac{A_{\mathbf{k}}}{\sqrt{A_{\mathbf{k}}^{2}-B_{\mathbf{k}}^{2}}}-1\right)\right] .
$$

In the general case correction terms in (5) and (6) should be evaluated by numerical integration over the three-dimensional Brillouin zone. However, analytical expressions representing the leading logarithmic terms can be found in the case of quasi one-dimensional magnets. Let us calculate them for hexagonal $\mathrm{CsNiCl}_{3}$-type antiferromagnets. Using the rectangular coordinate system, it is convenient to measure wave-vector components along the $z$ and $x$ axes in units of the reciprocal intersite distances $c$ and $a$ respectively, while the component in the $y$ direction in units of $2 / \sqrt{3} a$. Then, the Fourier transform of the exchange energy takes the form

$$
J_{\mathbf{k}}=2 J \cos k_{z}+6 J^{\prime} \gamma_{\mathbf{k}}, \quad \gamma_{\mathbf{k}}=\frac{1}{3}\left(\cos k_{x}+2 \cos \frac{1}{2} k_{x} \cos k_{y}\right)
$$

with each wave-vector component varying between 0 and $2 \pi$. The helix vector of the triangular structure found by minimization of $J_{\mathbf{k}}$ is $\mathbf{Q}=(4 \pi / 3,0, \pi)$ in this coordinate frame.

Keeping only the leading contributions in the quasi one-dimensional limit $J \gg J^{\prime}, D$, we omit terms proportional to $J^{\prime}$ and $D$ in numerators of Eqs. (5) and (6) and otbtain the following expressions $\left(H_{s} \approx 8 J S\right)$ : 


$$
\begin{gathered}
m=M / N=\frac{H}{8 J}\left[1-\frac{1}{2 S} \int \frac{d^{3} k}{(2 \pi)^{3}} \frac{\left(-\cos k_{z}\right) \sqrt{1-\cos k_{z}}}{\sqrt{1+\cos k_{z}+p_{\mathbf{k}}-H^{2} /\left(32 J^{2} S^{2}\right) \cos k_{z}}}\right] \\
S_{\mathrm{av}}=S\left[1-\frac{1}{2 S} \int \frac{d^{3} k}{(2 \pi)^{3}}\left(\frac{1-H^{2} /\left(64 J^{2} S^{2}\right) \cos k_{z}}{\sqrt{\left(1-\cos k_{z}+q_{\mathbf{k}}\right)\left(1+\cos k_{z}+p_{\mathbf{k}}-H^{2} /\left(32 J^{2} S^{2}\right) \cos k_{z}\right)}}-1\right)\right],
\end{gathered}
$$

where

$$
p_{\mathbf{k}}=\frac{3 J^{\prime}}{2 J}\left(1+2 \gamma_{\mathbf{k}}\right)+\frac{D}{2 J}, \quad q_{\mathbf{k}}=\frac{3 J^{\prime}}{2 J}\left(1-\gamma_{\mathbf{k}}\right) .
$$

The integration over $k_{z}$ in (8) can be done explicitly for arbitrary $p_{\mathbf{k}}$ and $H$. However, this would be an overestimation as we have already neglected linear and higher order terms in $J^{\prime} / J$. Therefore at $H \ll H_{s}$ we integrate (8) keeping only logarithmic and zero order in $p_{\mathbf{k}}$ terms. This yields:

$$
m=\frac{H}{8 J}\left\{1-\frac{1}{2 \pi S}\left[\ln \frac{16 J}{3 J^{\prime}}-2-\left\langle\ln \left(1+2 \gamma_{\mathbf{k}}+D / 3 J^{\prime}+H^{2} / 48 J J^{\prime} S^{2}\right)\right\rangle\right]\right\},
$$

where angular brackets denote averaging over the two-dimensional Brillouin zone. The sublattice magnetization is given with the same accuracy $O\left[\left(J^{\prime} / J\right) \ln \left(J / J^{\prime}\right)\right]$ by

$$
S_{\mathrm{av}}=S\left\{1-\frac{1}{2 \pi S}\left[\ln \frac{16 J}{3 J^{\prime}}-\pi-\frac{1}{2}\left\langle\ln \left(1-\gamma_{\mathbf{k}}\right)\right\rangle-\frac{1}{2}\left\langle\ln \left(1+2 \gamma_{\mathbf{k}}+D / 3 J^{\prime}+H^{2} / 48 J J^{\prime} S^{2}\right)\right\rangle\right]\right\} .
$$

The main part of quantum effects for both $m$ and $S_{\mathrm{av}}$ is represented at $H=0$ by the large logarithm $\ln \left(16 J / 3 J^{\prime}\right)$, which is in agreement with a qualitative suggestion of Ref. [15]. Numerical constants yield in the isotropic case only small corrections:

$$
\left\langle\ln \left(1+2 \gamma_{\mathbf{k}}\right)\right\rangle=-0.452, \quad\left\langle\ln \left(1-\gamma_{\mathbf{k}}\right)\right\rangle=-0.176
$$

At low fields the magnetization is a linear function of $H$ with the susceptibility per site

$$
\chi_{\perp}=\frac{1}{8 J}\left\{1-\frac{1}{2 \pi S}\left[\ln \frac{16 J}{3 J^{\prime}}-2-\left\langle\ln \left(1+2 \gamma_{\mathbf{k}}+D / 3 J^{\prime}\right)\right\rangle\right]\right\} .
$$

The additional correction terms in (10) and (11), however, grow with increasing field and give nonlinear dependences of magnetization and spin length. This nonlinear increase of $m$ and $S_{\text {av }}$ means suppression of quantum fluctuations in magnetic field, which must completely disappear in the collinear phase at $H \geq H_{s}$. 
In the isotropic case Eqs. (11) and (12) give good coincidence up to 2-3\% with the results by Ohyama and Shiba [11], who estimated integrals in (5) and (6) numerically. Note, that all the derived expressions are also valid for the easy-axis helimagnet at fields above the spin-flop field $(\mathbf{H} \| \hat{\mathbf{z}})$, when spins lie in the basal plane and the axial symmetry is restored. In this case the constant $D$ in (10) and (11) has the negative sign as in the Hamiltonin (1).

At high fields the magnitude of quantum corrections to $m$ becomes independent of the interchain coupling. Crossover between two regimes takes place at a characteristic field $H_{c}=\sqrt{48 J^{\prime} J S^{2}}$. At $H_{c} \ll H \leq H_{s}$ one can neglect the term $p_{\mathbf{k}}$ in (8) and obtain after integration over $k_{z}$ the magnetization

$$
\begin{gathered}
m=S \frac{H}{H_{s}}\left[1-\frac{1}{\pi S} \frac{H_{s} \sqrt{H_{s}^{2}-H^{2}}}{2 H^{2}-H_{s}^{2}}\left(1-\sqrt{\frac{H_{s}^{2}-H^{2}}{\mid 2 H^{2}-H_{s}^{2}}} f(H)\right)\right], \\
\text { where } f(H)= \begin{cases}\ln \left(\sqrt{\frac{H_{s}^{2}}{H^{2}}-2}+\sqrt{\frac{H_{s}^{2}}{H^{2}}-1}\right), & H \leq H_{s} / \sqrt{2}, \\
\arcsin \sqrt{2-\frac{H_{s}^{2}}{H^{2}}}, & H_{s} / \sqrt{2} \leq H \leq H_{s} .\end{cases}
\end{gathered}
$$

As for the staggered magnetization $S_{\mathrm{av}}$, it remains finite in the field region $0<H<H_{s}$ only if $J^{\prime} \neq 0$.

According to (13) the magnetization approaches its saturation value with the asymptotic behavior $M=M_{s}\left[1-\sqrt{2\left(1-H / H_{s}\right)} / \pi S\right]$. As we neglected the interchain coupling, this result should be also valid for a single antiferromagnetic chain. Hodgson and Parkinson [3] using the Bethe ansatz approach found a different critical behavior for the chain magnetization:

$$
M=M_{s}\left[1-2 \sqrt{1-H / H_{s}} / \pi S\right] .
$$

The reason of this discrepancy can be explained as follows. In the bosonic representation the Hamiltonian (1) is equivalent to a gas of Bose particles interacting via a $\delta$-potential. Their interaction proportional to $1 / S$ is neglected in the harmonic approximation. As magnetic field approaches the saturation field, the boson density tends to zero. In the low-density limit the average kinetic energy of bosons is small and they behave as if the potential has a hard core. Therefore, in one dimension at fields sufficiently close to $H_{s}$ the interaction between spin waves becomes non-negligible for any value of $S$ and gives the modified asymptotic behavior [6]. The width of the critical region of quantum fluctuations decreases with increasing $S$. 
We compare our expression for the magnetization (13) with the results of exact numerical diagonalization of the Hamiltonian for finite systems. As can be seen from Fig. 1, the formula (13) is in remarkable agreement with the magnetization curve for a spin-1 antiferromagnetic chain found numerically by Sakai and Takahashi [5] except for a low-field region where the nonmagnetic Haldane phase is developed and a close vicinity of $H_{s}$. Thus, we conclude that at the intermediate field region the magnetic field suppresses zero-point fluctuations and increases the accuracy of the spin-wave theory. The magnetization curve for a system of coupled antiferromagnetic chains does not depend at high fields on the nature of the ground state (which is determined by $J^{\prime}$ and $S$ ) and is given by (13). However, in the narrow field region near $H_{s}$ the interaction between spin-wave modes becomes important and changes the asymptotic behavior of $M(H)$. The size of this region is small already for $S=1$ and the harmonic approximation of the spin-wave theory gives good account for most of the experimental results at high fields $[1,2,18]$.

\section{IN-PLANE ANISOTROPY}

Consider further quantum effects for a Heisenberg helimagnet with an in-plane anisotropy. The easy-axis anisotropy or magnetic field applied in the spin plane perturb spin helix adding higher-order harmonics $n \mathbf{Q}[21,22]$. Derivations of quantum corrections are similar for all cases. We present in detail calculations for $D<0$ and $H=0$ listing in other cases only final expressions.

If the anisotropy constant $D$ in (1) is negative, spins in the spiral structure rotate in the vertical plane: $\mathbf{S}_{i}=\left(S \sin \theta_{i}, 0, S \cos \theta_{i}\right)$. As the exchange interactions are much stronger than the relativistic anisotropy we can consider only the first anharmonic term

$$
\theta_{i}=\mathbf{Q} \cdot \mathbf{r}_{i}-\varphi \sin \left(2 \mathbf{Q} \cdot \mathbf{r}_{i}\right)
$$

Making substitution of (15) into (1), we find the following expression for the deviation angle:

$$
\varphi=|D| /\left(J_{3 \mathbf{Q}}-J_{\mathbf{Q}}\right) .
$$

The formula (15) is valid for both incommensurate and commensurate antiferromagnets (if only $3 \mathbf{Q} \neq \pm \mathbf{Q}$ ). In the latter case there should be an additional constant term in (15) which describes lock-in of a spin helix with respect to the crystal axes. However, we can neglect 
it, because the commensuration energy appears only at the $N / 2$ order of the expansion in $|D|$, where $N \mathbf{Q}=\mathbf{G}, \mathbf{G}$ being the reciprocal lattice vector [22].

Taking into account (15) the quadratic part of the Hamiltonian is written as

$$
\begin{aligned}
\hat{\mathcal{H}}= & -\frac{1}{4} N|D| S+\sum_{\mathbf{k}}\left[A_{\mathbf{k}} a_{\mathbf{k}}^{+} a_{\mathbf{k}}+\frac{1}{2} B_{\mathbf{k}}\left(a_{\mathbf{k}} a_{-\mathbf{k}}+a_{\mathbf{k}}^{+} a_{-\mathbf{k}}^{+}\right)\right. \\
& \left.+C_{\mathbf{k}}\left(a_{\mathbf{k}+\mathbf{Q}}^{+} a_{\mathbf{k}-\mathbf{Q}}+\text { c.c. }\right)+\frac{1}{2} D_{\mathbf{k}}\left(a_{\mathbf{k}+\mathbf{Q}} a_{-\mathbf{k}+\mathbf{Q}}+a_{\mathbf{k}-\mathbf{Q}} a_{-\mathbf{k}-\mathbf{Q}}+\text { c.c. }\right)\right],
\end{aligned}
$$

where

$$
\begin{aligned}
& A_{\mathbf{k}}=-2 J_{\mathbf{Q}} S+S\left[J_{\mathbf{k}}+\frac{1}{2}\left(J_{\mathbf{k}+\mathbf{Q}}+J_{\mathbf{k}-\mathbf{Q}}\right)\right]+\frac{1}{2}|D| S, \\
& B_{\mathbf{k}}=-S\left[J_{\mathbf{k}}-\frac{1}{2}\left(J_{\mathbf{k}+\mathbf{Q}}+J_{\mathbf{k}-\mathbf{Q}}\right)\right]-\frac{1}{2}|D| S, \\
& C_{\mathbf{k}}=\frac{5}{4}|D| S+\frac{1}{2} \varphi S\left[J_{\mathbf{k}}-\frac{1}{2}\left(J_{\mathbf{k}+2 \mathbf{Q}}+J_{\mathbf{k}-2 \mathbf{Q}}\right)\right], \quad D_{\mathbf{k}}=C_{\mathbf{k}}-|D| S .
\end{aligned}
$$

As was shown by Elliot and Lange using general symmetry arguments [23], a helimagnet with in-plane magnetic field always have a zero-frequency mode for spin rotations inside helix plane. Their theorem fulfills obviously for another types of in-plane anisotropy. We illustrate this by finding in the first order in $D$ analytical expressions for the classical spectrum and the ground state energy of an easy-axis helimagnet.

The Bogolyubov transformation can be used to diagonalize the first two terms in (16). After this procedure the boson part of the Hamiltonian (16) is reduced to

$$
\begin{aligned}
\hat{\mathcal{H}}= & \sum_{\mathbf{k}}\left[\omega_{\mathbf{k}}^{0}\left(b_{\mathbf{k}}^{+} b_{\mathbf{k}}+\frac{1}{2}\right)-\frac{1}{2} A_{\mathbf{k}}+\tilde{C}_{\mathbf{k}}\left(b_{\mathbf{k}+\mathbf{Q}}^{+} b_{\mathbf{k}-\mathbf{Q}}+\text { c.c. }\right)\right. \\
& \left.+\frac{1}{2} \tilde{D}_{\mathbf{k}}\left(b_{\mathbf{k}+\mathbf{Q}} b_{-\mathbf{k}+\mathbf{Q}}+b_{\mathbf{k}-\mathbf{Q}} b_{-\mathbf{k}-\mathbf{Q}}+\text { c.c. }\right)\right],
\end{aligned}
$$

where

$$
\begin{aligned}
& \omega_{\mathbf{k}}^{0}=\sqrt{A_{\mathbf{k}}^{2}-B_{\mathbf{k}}^{2}}, \quad \tanh 2 \psi_{\mathbf{k}}=-B_{\mathbf{k}} / A_{\mathbf{k}}, \\
& \tilde{C}_{\mathbf{k}}=C_{\mathbf{k}} \cosh \left(\psi_{\mathbf{k}+\mathbf{Q}}+\psi_{\mathbf{k}-\mathbf{Q}}\right)+D_{\mathbf{k}} \sinh \left(\psi_{\mathbf{k}+\mathbf{Q}}+\psi_{\mathbf{k}-\mathbf{Q}}\right), \\
& \tilde{D}_{\mathbf{k}}=C_{\mathbf{k}} \sinh \left(\psi_{\mathbf{k}+\mathbf{Q}}+\psi_{\mathbf{k}-\mathbf{Q}}\right)+D_{\mathbf{k}} \cosh \left(\psi_{\mathbf{k}+\mathbf{Q}}+\psi_{\mathbf{k}-\mathbf{Q}}\right) .
\end{aligned}
$$

Note, that constants $\omega_{\mathbf{k}}^{0}, A_{\mathbf{k}}$ and $B_{\mathbf{k}}$ are given by the same expressions as in the case of the easy-plane anisotropy (2) but with $D_{\text {eff }}=|D| / 2$. Attempt to find eigenfrequencies of (17) leads to an infinite system of coupled equations. However, the diagonalization may be simplified since the amplitudes $\tilde{C}_{\mathbf{k}}, \tilde{D}_{\mathbf{k}}$ are of the order of $|D| S$ everywhere in the Brillouin zone except for vicinity of the point $\mathbf{k}=0$, where the Bogolyubov transformation becomes nonanalytical in the limit $|D| \rightarrow 0$. Therefore umklapp terms in the Hamiltonian (17) 
are important for the calculation of the spin-wave spectrum in the first order in $|D| S$ only near $\mathbf{k}= \pm \mathbf{Q}$, where they describe interaction of four nearly degenerate magnons with propagation vectors $\pm \mathbf{Q} \pm \mathbf{q}$.

In the following calculations we use the method proposed by White [24]. We define the column vector of bose operators $\hat{x}_{\mathbf{q}}=\left(b_{\mathbf{q}+\mathbf{Q}}, b_{\mathbf{q}-\mathbf{Q}}, b_{-\mathbf{q}+\mathbf{Q}}^{+}, b_{-\mathbf{q}-\mathbf{Q}}^{+}\right)$. Then, excluding the constant term $-\frac{1}{2} \sum_{\mathbf{k}} A_{\mathbf{k}}$, the Hamiltonian (17) can be rewritten as

$$
\hat{\mathcal{H}}=\sum_{\mathbf{q}} \hat{x}_{\mathbf{q}}^{+} \mathcal{H}_{\mathbf{q}} \hat{x}_{\mathbf{q}}
$$

where

$$
\mathcal{H}_{\mathbf{q}}=\left(\begin{array}{cccc}
\omega_{\mathbf{q}+\mathbf{Q}}^{0} & \tilde{C}_{\mathbf{q}} & 0 & \tilde{D}_{\mathbf{q}} \\
\tilde{C}_{\mathbf{q}} & \omega_{\mathbf{q}-\mathbf{Q}}^{0} & \tilde{D}_{\mathbf{q}} & 0 \\
0 & \tilde{D}_{\mathbf{q}} & \omega_{\mathbf{q}+\mathbf{Q}}^{0} & \tilde{C}_{\mathbf{q}} \\
\tilde{D}_{\mathbf{q}} & 0 & \tilde{C}_{\mathbf{q}} & \omega_{\mathbf{q}-\mathbf{Q}}^{0}
\end{array}\right)
$$

Following the above arguments, we replace the eigenvalue problem for the infinite matrix $\hat{\mathcal{H}}$ by diagonalization of $4 \times 4$ matrix (19). The transformation $\hat{x}_{\mathbf{k}}=S \hat{y}_{\mathbf{k}}$ that diagonalizes $\mathcal{H}_{\mathbf{q}}$ must preserve the commutator $\left[\hat{x}_{\mathbf{k}}, \hat{x}_{\mathbf{k}}^{+}\right]=g$, where the metric matrix $g$ has only diagonal elements: $(+1,+1,-1,-1)$. Solving equation

$$
S^{-1} g \mathcal{H}_{\mathbf{q}} S=g \Omega_{H}
$$

we obtain the diagonal matrix $\Omega_{H}$ with two pairs of equal eigenfrequencies

$$
\begin{aligned}
\left(\omega^{ \pm}\right)^{2}= & \frac{1}{2}\left[\left(\omega_{\mathbf{q}+\mathbf{Q}}^{0}\right)+\left(\omega_{\mathbf{q}-\mathbf{Q}}^{0}\right)^{2}\right]+\tilde{C}_{\mathbf{q}}^{2}-\tilde{D}_{\mathbf{q}}^{2} \pm\left\{\frac{1}{4}\left[\left(\omega_{\mathbf{q}+\mathbf{Q}}^{0}\right)^{2}-\left(\omega_{\mathbf{q}-\mathbf{Q}}^{0}\right)^{2}\right]^{2}\right. \\
& \left.+\tilde{C}_{\mathbf{q}}^{2}\left(\omega_{\mathbf{q}+\mathbf{Q}}^{0}+\omega_{\mathbf{q}-\mathbf{Q}}^{0}\right)^{2}-\tilde{D}_{\mathbf{q}}^{2}\left(\omega_{\mathbf{q}+\mathbf{Q}}^{0}-\omega_{\mathbf{q}-\mathbf{Q}}^{0}\right)^{2}\right\}^{1 / 2}
\end{aligned}
$$

which can be mapped onto two different magnon branches in a half of the Brillouin zone. At $\mathbf{k}=\mathbf{Q}$ the frequency gap of the first branch is $\omega_{\mathbf{Q}}^{+}=2 S\left[|D|\left(J_{0}+J_{2 \mathbf{Q}}-2 J_{\mathbf{Q}}\right)\right]^{1 / 2}$, while $\omega_{\mathbf{Q}}^{-}=0$. Thus, the classical spectrum of a helimagnet with the easy-axis anisotropy contains two quasi Goldstone modes at $\mathbf{k}=0$ and $\mathbf{Q}$, where the additional zero-gap mode results from the absence of the basal plane anisotropy in the Hamiltonian (1). We emphasize again that this conclusion is valid for a commensurate helimagnet only in the first order in $|D|$. For example, the second mode at $\mathbf{k}=\mathbf{Q}$ in triangular antiferromagnets acquires a frequency gap proportional to $|D|^{3 / 2}[22]$. 
The energy of zero-point fluctuations is determined after the last diagonalization by $E^{(0)}=\frac{1}{2} \sum_{\mathbf{k}>\mathbf{0}}\left(\omega_{\mathbf{k}}^{+}+\omega_{\mathbf{k}}^{-}\right)$. However, the umklapp terms in (17) yield correction to the energy of the ground state only in the second order in anisotropy constant and, therefore, should be omitted. The ground state energy in the first order in $|D|$ can be obtained after partial diagonalization:

$$
E_{\text {g.s. }}=J_{\mathbf{Q}} S(S+1)-\frac{1}{2}|D| S(S+1)+\frac{1}{2} \sum_{\mathbf{k}} \omega_{\mathbf{k}}^{0} .
$$

To calculate the staggered magnetization with the same accuracy one has, in contrast, to determine explicitly the transformation matrix $S$. Expressing operators $a_{i}$ via new boson modes, it is possible to find the average spin length including its modulation $\left[\sim \cos \left(2 \mathbf{Q} \cdot \mathbf{r}_{i}\right)\right]$ in a bunched spiral. These calculations cannot be done analytically and require numerical study which was presented in the case of triangular antiferromagnets by Watabe et al. [20]. Note, that for a small anisotropy (the case with which we are only concerned here) the sublattice magnetization can be roughly estimated as in the isotropic case by using Eq. (11) with $D=0$.

Expanding (22) up to the first order in $D$, we get the anisotropy energy of a triangular antiferromagnet renormalized by zero-point oscillations

$$
E_{\text {an }}^{(1)}=-\frac{|D| S(S+1)}{2}+\frac{|D| S}{4 \pi}\left[\ln \frac{16 J}{3 J^{\prime}}-\left\langle\ln \left(1+2 \gamma_{\mathbf{k}}\right)\right\rangle\right] .
$$

If magnetic field is applied along the easy axis, the vertical position of the helix plane becomes unstable at high fields. Magnetic energy exceeds the relativistic anisotropy, and above the spin-flop field $H_{\mathrm{sf}}$ spins lie in the basal plane. In the classical picture, anisotropic part of the energy for this configuration $E_{\text {an }}^{(2)}$ vanishes. However, zero-point motion of quantum spins makes $E_{\mathrm{an}}^{(2)} \neq 0$. Expanding the ground state energy (3) in powers of $D$, we have in the first order

$$
E_{\mathrm{an}}^{(2)}=-\frac{|D| S}{2 \pi}\left[\ln \frac{16 J}{3 J^{\prime}}-\left\langle\ln \left(1+2 \gamma_{\mathbf{k}}\right)\right\rangle\right] .
$$

The energy difference for vertical and horizontal configurations of polarization plane $E_{\text {an }}=$ $E_{\text {an }}^{(2)}-E_{\text {an }}^{(1)}$ will be used below for the calculation of the spin-flop field.

Consider now the effect of magnetic field applied along the easy axis. The distortion of a spin helix is described in this case by

$$
\theta_{i}=\mathbf{Q} \cdot \mathbf{r}_{i}-\alpha \sin \left(\mathbf{Q} \cdot \mathbf{r}_{i}\right)-\varphi \sin \left(2 \mathbf{Q} \cdot \mathbf{r}_{i}\right)
$$


with $\alpha=H /\left(J_{0}+J_{2 \mathbf{Q}}-2 J_{\mathbf{Q}}\right) S[21,22]$. As we explained above, the spin harmonics generated by single-ion anisotropy, being important for calculations of the correct classical spin-wave spectrum, can be neglected for the estimate of the ground state energy in the first order in $D$. For the latter it is necessary to take into account only changes in constants $A_{\mathbf{k}}$ and $B_{\mathbf{k}}$. The new spin harmonic generated by magnetic field gives

$$
\delta A_{\mathbf{k}}=\delta B_{\mathbf{k}}=-\frac{1}{4} \alpha^{2} S\left[J_{\mathbf{k}-\mathbf{Q}}+J_{\mathbf{k}+\mathbf{Q}}-J_{\mathbf{k}}-\frac{1}{2}\left(J_{\mathbf{k}-2 \mathbf{Q}}+J_{\mathbf{k}+2 \mathbf{Q}}\right)\right] .
$$

Magnetic field also generates umklapp terms in the boson Hamiltonian with a momentum transfer $\pm \mathbf{Q}$. These terms are proportional to $\alpha$ and, therefore, should be taken into account for calculation of the ground state energy with the accuracy $O\left(\alpha^{2}\right) \sim H^{2}$. Inspection of corresponding expressions reveals, however, that umklapp terms have an additional smallness of the order $J^{\prime} / J$ in the quasi one-dimensional limit. Having in mind this case, one can calculate magnetic contribution to the ground state energy without umklapp terms as in the above analysis of the easy-axis anisotropy. The uniform magnetization found in this approximation is

$$
m=\frac{H}{2\left(J_{0}+J_{2 \mathbf{Q}}-2 J_{\mathbf{Q}}\right)}\left[1-\frac{1}{2 S N} \sum_{\mathbf{k}} \frac{J_{\mathbf{k}}+\frac{1}{2}\left(J_{\mathbf{k}+2 \mathbf{Q}}+J_{\mathbf{k}-2 \mathbf{Q}}\right)-J_{\mathbf{k}-\mathbf{Q}}-J_{\mathbf{k}+\mathbf{Q}}}{J_{0}+J_{2 \mathbf{Q}}-2 J_{\mathbf{Q}}} \sqrt{\frac{A_{\mathbf{k}}-B_{\mathbf{k}}}{A_{\mathbf{k}}+B_{\mathbf{k}}}}\right] .
$$

Analytical estimate of the integral in (26) yields the following expression for the in-plane susceptibility of a quasi one-dimensional triangular antiferromagnet

$$
\chi_{\|}=\frac{1}{16 J}\left\{1-\frac{1}{2 \pi S}\left[\ln \frac{16 J}{3 J^{\prime}}-2-\left\langle\ln \left(1-\gamma_{\mathbf{k}}\right)\right\rangle\right]\right\} .
$$

Note, that quantum corrections break the relation $\chi_{\perp}=\chi_{\|}$, which holds for a classical antiferromagnetic helimagnet in the quasi one-dimensional limit.

With the help of (12), (23), (24), and (27) we find the critical field of the spin-flop transition as a field at which anisotropy and magnetic energies of a quantum helimagnet are equal:

$$
H_{\mathrm{sf}}^{2}=16 D J S^{2} \frac{1+1 / S-3 /(2 \pi S)\left[\ln \left(16 J / 3 J^{\prime}\right)-\left\langle\ln \left(1+2 \gamma_{\mathbf{k}}\right)\right\rangle\right]}{1-1 /(2 \pi S)\left[\ln \left(16 J / 3 J^{\prime}\right)-2-2\left\langle\ln \left(1+2 \gamma_{\mathbf{k}}\right)\right\rangle+\left\langle\ln \left(1-\gamma_{\mathbf{k}}\right)\right\rangle\right]} .
$$

Let us list at the end the results for two another important cases, which can be derived in a similar way: (i) $D<0, \mathbf{H} \perp \hat{\mathbf{z}}$, the magnetization is given by the formula for the easy-plane anisotropy (10) with the substitution $D \rightarrow|D| / 2$; (ii) $D>0, \mathbf{H} \perp \hat{\mathbf{z}}$, in-plane susceptibility in this case coincides with (27). 


\section{DISCUSSION}

We compare our theoretical results with measurements on two isostructural quasi onedimensional hexagonal antiferromagnets $\mathrm{CsNiCl}_{3}$ and $\mathrm{CsMnBr}_{3}$, for which a lot of experimental data are available including the zero-point spin reductions $[12,13]$ and precise magnetization curves $[14,15]$. Both compounds are considered to be ideal Heisenberg systems described by the Hamiltonian (1) and differ only in sign and relative strength of the anisotropy constants.

First we consider the case of $\mathrm{CsMnBr}_{3}$, which has a large spin $(S=5 / 2)$ and the easy-plane type of anisotropy. The following values of the constants in the Hamiltonian (1) were obtained by fitting the measured magnon frequencies to the classical spin-wave dispersion [13,14]: $J=214 \mathrm{GHz}, J^{\prime}=0.5 \mathrm{GHz}, D=1.95 \mathrm{GHz}$, and $g=2$. It should be mentioned here that the magnon spectrum is also subjected to quantum renormalizations. If they are not too large the functional dependences of the magnon frequencies are expected to preserve the classical form, which involve, however, renormalized values $\tilde{J}, \tilde{J}^{\prime}$, and $\tilde{D}$. Major renormalizations in this case are found to be [25]

$$
\tilde{J}=J\left(1+\frac{\pi-2}{2 \pi S}\right), \quad \tilde{D}=D\left(1-\frac{1}{2 S}\right) .
$$

For $S=5 / 2$ these corrections are not so important and one can use the above set of constants

to fit the experiment. With the help of (11) we obtain the average value of spin $S_{\mathrm{av}}=$ 1.82, which is not far from the experimental value $S_{\mathrm{av}}^{\exp }=1.65$ [13]. However, the notable discrepancy about $10 \%$ is quite surprising. It cannot be explained by the renormalization of constants (29) and shows the limits of the harmonic approximation to describe a large spin reduction even for $S=5 / 2$. Next we calculate the magnetization curve for $\mathbf{H} \| \hat{\mathbf{z}}$ using the expression (10). The result is presented in Fig. 2 (solid line) together with the experimental data [14]. Theoretical curve coincides nicely with the experiment reproducing the unusually low value of the susceptibility and a small upturn curvature of the magnetization. Better agreement between the theory and experiment for the uniform magnetization than for the average spin value is clearly expected for the quasi one-dimensional antiferromagnet with a large easy-plane anisotropy $D>J^{\prime}$. In the one-dimensional limit $\left(J^{\prime}=0\right)$ the average spin value calculated in the linear spin-wave theory diverges showing its inapplicability, while the magnetization (10) remains to be finite. For $\mathbf{H} \perp \hat{\mathbf{z}}$ we calculate only the slope of the magnetization curve using the expression (27) for the susceptibility. The resultant curve 
(dashed line in Fig. 2) again coincides well with the experiment reproducing the quantum reduction of the susceptibility. At higher fields for this orientation of $\mathbf{H}$ the distortions of the triangular structure become important which requires to take into account the umklapp terms. At $H>H_{c}$ the spin structure transforms into a collinear one [14].

Next we consider $\mathrm{CsNiCl}_{3}$ which has the easy-axis type of anisotropy and much more pronounced quantum effects due to $S=1$. In order to calculate the magnetization for $\mathbf{H} \perp \hat{\mathbf{z}}$ we use the expressions (10) with $D_{\text {eff }}=|D| / 2$. For $\mathbf{H} \| \hat{\mathbf{z}}$ the magnetization is given by (26) below the spin-flop transition and by (10) but with negative $D$ above $H_{\text {sf }}$. In the case of $\mathrm{CsNiCl}_{3}$ there is a serious problem to determine values of the constants in the Hamiltonian (1) from the spin-wave dispersions since the classical expression (22) does not reproduce the dependences observed on the experiment [8]. The discrepancy is small for the magnon dispersion along chains, and one can estimate from the zone boundary energy $\tilde{J}=345 \mathrm{GHz}[8]$. With this value of $\tilde{J}$ the fit of the low-frequency magnetic resonance data and the spin-flop field value to the classical expressions yield $\tilde{J}^{\prime}=8 \mathrm{GHz}$ and $\tilde{D}=-0.6 \mathrm{GHz}$ [26]. If we calculate the magnetization curves using this set of parameters, our results show rather poor agreement with the experimental data. Large part of the error comes from the use of the renormalized values for $J, J^{\prime}$, and $D$ instead of the bare Hamiltonian constants. For $S=1$ the renormalizations are quite important: according to (29) the bare value of $J$ in this case is about $18 \%$ smaller than $\tilde{J}$, while $D$ is twice larger than $\tilde{D}$.

On the other hand, the bare value of the exchange constant $J$ can be determined from the spin-flip field $H_{s}$ which is not renormalized due to the absence of quantum fluctuations in the saturated phase at $H>H_{s}$. Recent high-field magnetization measurements [18] gave $J=275 \mathrm{GHz}$ and $g=2.15$. We used these values together with $J^{\prime}=8 \mathrm{GHz}$ and $D=-1.6 \mathrm{GHz}$ to calculate theoretical curves which are shown in Fig. 3. The choice of the bare anisotropy constant is rather arbitrary since it cannot be calculated from the spin-flop field: for such large value of the ratio $J / J^{\prime}$ Eq. (28) fails due to the negative sign of the right hand side. Instead, we have chosen the value of $D$ that gives reasonable description of the difference of magnetizations for the two perpendicular orientations of magnetic field at $H>H_{\mathrm{sf}}$. Note, that this difference is connected with a zero-point contribution to the anisotropy energy, analogous to the case of $\mathrm{CsMnBr}_{3}$ above $H_{c}[14,16]$. Our calculations with the above constants presented in Fig. 3 give adequate values of the susceptibilities but distinct difference in the field dependence of the magnetization. We note that the 
disagreement between the classical spin-flop field depicted in the Figure and the experimental value does not make any sense since both the classical $\sqrt{16 D J S^{2}}$ and the renormalized (28) expressions for $H_{\mathrm{sf}}$ are inapplicable for $\mathrm{CsNiCl}_{3}$. This fact together with a poor agreement of the nonlinear growth of the magnetization point to the insufficiency of the harmonic approximation and need to develop a more accurate treatment including higher-order terms in $1 / S$. Finally, the sublattice magnetization calculated neglecting the single-ion anisotropy is $S_{\mathrm{av}}=0.62$ compared with the experimental value $S_{\mathrm{av}}^{\exp }=0.52$.

\section{CONCLUSIONS}

We calculated the leading contribution of zero-point fluctuations to the static properties of a quasi one-dimensional antiferromagnetic helimagnet. Use of the symmetry of the spin structure for $D>0, \mathbf{H} \| \hat{\mathbf{z}}$ and perturbative treatment of the anisotropy for $D<0$ allowed us to develop a spin-wave theory with only one kind of boson operators in the paramagnetic Brillouin zone and to obtain simple analytic expressions for quantum effect. Note, that the traditional approach in the framework of the multisublattice model leads to large mathematical complications and does not yield analytical results [20], though it should be applied in the case of a strong Ising-like anisotropy.

Comparison with the experiment show that theory give good qualitative description of all specific features observed in the real quasi one-dimensional antiferromagnets. They include: strong reduction of the ordered moments and the susceptibility, nonlinear growth of the transverse magnetization and its anisotropy in the easy-axis case above $H_{\mathrm{s}-\mathrm{f}}$. Accurate quantitative comparison between the theory and experiment is difficult and give ambiguous results since the bare constants involved in the Hamiltonian (1) are not known.

Zero-point fluctuations also affect in a different way different magnetic characteristics. The calculated correction to the spin-flop field makes its value imaginary in the first order in $1 / S$ for $S=1$ what signals about importance of higher-order corrections. Instead, the results of the harmonic approximation give quite good description of the magnetization curve at high fields even for a single chain. Corresponding expression (13) fails only in the close neighborhood of the spin-flip field $H_{s}$ where it should be corrected via account of the magnon interactions. 


\section{ACKNOWLEDGMENTS}

M.E.Zh. thanks the Japan Society for the Promotion of Science for partial financial

support on this work. The work of I. A.Z. was supported by the Grant M3K000 of the International Science Foundation, Moscow and by the INTAS Grant N93-1107. 


\section{REFERENCES}

[1] K. Katsumata, H. Hori, T. Takeuchi, M. Date, A. Yamadgishi, and J. P. Renard, Phys. Rev. Lett. 63, 86 (1989); Y. Ajiro, T. Goto, H. Kikuchi, T. Sakakibara, and T. Inami, ibid. 63, 1424 (1989).

[2] H. Nojiri, Y. Shimamoto, N. Miura, and Y. Ajiro, J. Phys.: Cond. Matt. 7, 5881 (1995).

[3] J. B. Parkinson and J. C. Bonner, Phys. Rev. B 32, 4703 (1985); R. P. Hodgson and J. B. Parkinson, J. Phys. C 18, 6385 (1985).

[4] I. Affleck, Phys. Rev. B 41, 6697 (1990); 43, 3215 (1991).

[5] T. Sakai and M. Takahashi, Phys. Rev. B 43, 13383 (1991).

[6] M. Takahashi and T. Sakai, J. Phys. Soc. Jpn. 60, 760 (1991).

[7] S. Yamamoto and S. Miyashita, Phys. Rev. B 51, 3649 (1995).

[8] W. J. L. Buyers, R. M. Morra, R. L. Armstrong, M. J. Hogan, P. Gerlach, and K. Hirakawa, Phys. Rev. Lett. 56, 371 (1986); R. M. Morra, W. J. L. Buyers, R. L. Armstrong, and K. Hirakawa, Phys. Rev. B 38, 543 (1988).

[9] A. V. Chubukov, J. Phys. C 17, L991 (1984); E. Rastelli, L. Reatto, and A. Tassi, ibid. 18, $353(1985)$.

[10] I. Affleck, Phys. Rev. Lett. 62, 474 (1989); M. L. Plumer and A. Caillé, ibid. 68, 1042 (1992); I. Affleck and G. F. Wellman, Phys. Rev. B 46, 8934 (1992).

[11] T. Ohyama and H. Shiba, J. Phys. Soc. Jpn. 62, 3277 (1993); 63, 3454 (1994).

[12] W. B. Yelon and D. E. Cox, Phys. Rev. B 6, 204 (1972); 7, 2024 (1973).

[13] M. Eibshutz, R. C. Sherwood, F. S. L. Hsu, and D. E. Cox, AIP Conf. Proc. 17, 864 (1972).

[14] S. I. Abarzhi, A. N. Bazhan, L. A. Prozorova, and I. A. Zaliznyak, J. Phys.: Cond. Matt. 4, 3307 (1992).

[15] I. A. Zaliznyak, Solid State Commun. 84, 573 (1992).

[16] A. G. Abanov and O. A. Petrenko, Phys. Rev. B 50, 6271 (1994). 
[17] W. Palme, H. Kriegelstein, O. Born, A. Chernaoui, and B. Lüthi, Z. Phys. B 92, 1 (1993).

[18] H. A. Katori, Y. Ajiro, T. Asano, and T. Goto, preprint ISSP No. 2950, submitted to J. Phys. Soc. Jpn. (1995).

[19] D. Weltz, J. Phys.: Cond. Matt. 5, 3643 (1993).

[20] Y. Watabe, T. Suzuki, and Y. Natsume, Phys. Rev. B 52, 3400 (1995).

[21] T. Nagamiya, in Solid State Physics, edited by F. Seitz, D. Turnbull, and H. Ehrenreich (Academic Press, New York, 1967), Vol. 20, P. 305.

[22] I. A. Zaliznyak and M. E. Zhitomirsky, Zh. Eksp. Teor. Fiz. 108, 1052 (1995) [JETP 81, $568(1995)]$.

[23] R. J. Elliott and R. V. Lange, Phys. Rev. 152, 235 (1966).

[24] R. M. White, Quantum theory of magnetism (Springer, Berlin, 1983), P. 191.

[25] T. Oguchi, Phys. Rev. 117, 117 (1960); P.-A. Lindgård and A. Kowalska, J. Phys. C 9, 2081 (1976).

[26] I. A. Zaliznyak, L. A. Prozorova, and A. V. Chubukov, J. Phys.: Condens. Matter 1, 4743 (1989). 


\section{FIGURES}

FIG. 1. Magnetization curve for a spin-1 antiferromagnetic chain. Squares represent the numerical results of Ref. [5]. Solid line is the renormalized spin-wave theory prediction (13), dashed line is the magnetization asymptotics near the saturation field [3].

FIG. 2. Magnetization curves in $\mathrm{CsMnBr}_{3}$. Closed and open circles are experimental data per mole for $\mathbf{H} \perp \hat{\mathbf{z}}$ and $\mathbf{H} \| \hat{\mathbf{z}}$, respectively [14]. Solid line is calculated using Eq. (10) with the parameters $J=214 \mathrm{GHz}, J^{\prime}=0.5 \mathrm{GHz}$ and $D=1.95 \mathrm{GHz}$; dashed line is the low-field asymptotics with the same set of parameters.

FIG. 3. Magnetization curves in $\mathrm{CsNiCl}_{3}$. Closed and open circles are experimental data per

mole for $\mathbf{H} \perp \hat{\mathbf{z}}$ and $\mathbf{H} \| \hat{\mathbf{z}}$, respectively [15]. Solid lines are the renormalized spin-wave theory predictions with the parameters $J=275 \mathrm{GHz}, J^{\prime}=8 \mathrm{GHz}$ and $D=-1.6 \mathrm{GHz}$. 